\title{
Four-week inhalation toxicity study of 1-propanol in F344 rats
}

\author{
Yong-Soon Kim ${ }^{1}$ Eun-Sang Cho ${ }^{1} \cdot$ Ka-Young Park $^{1} \cdot$ Cheol-Hong Lim ${ }^{1}$
}

Received: 19 July 2019 / Revised: 5 November 2019 / Accepted: 27 November 2019 / Published online: 20 January 2020

(c) Korean Society of Toxicology 2020

\begin{abstract}
1-Propanol is used as a solvent for waxes, vegetable oils, resins, cellulose esters, and ethers, and is not considered harmful to humans by food and non-occupational exposures. However, workers are potentially exposed to 1-propanol by inhalation when it is used in the workplace. Thus, inhalation toxicity data are needed to assess the hazard of 1-propanol for workers safety. Five male and five female F344 rats were exposed to 1-propanol vapor for 4-weeks ( 6 h/day, 5 days/week) at concentrations of $0,100,400$, and $1600 \mathrm{ppm}$ in a whole-body inhalation chamber system. The actual exposure concentrations were $100.11 \pm 5.10,403.19 \pm 12.31$, and $1598.08 \pm 139.58 \mathrm{ppm}$ for the low, middle, and high dose groups, respectively. No clinical signs, significant mean body weight changes, significant changes of hematology or blood biochemistry results, or histopathological abnormalities were seen related to exposure to the test substance. Under the conditions of this study, the no-observed-adverse-effect level of 1-propanol was over $1600 \mathrm{ppm}$.
\end{abstract}

Keywords Inhalation toxicity $\cdot 1$-Propanol $\cdot$ No-observed-adverse-effect level $\cdot$ Occupational exposure

\section{Introduction}

1-Propanol is a colorless and volatile liquid with a sweet and pleasant odor. It occurs naturally in crude fossil fuels, and as a fermentation and decomposition product of various fruits and vegetables $[1,2]$. This substance is used as a solvent for waxes, vegetable oils, resins, cellulose esters, and ethers. It is also found in inks, brake fluids, and polishing compounds. The United States Environmental Protection Agency expects that exposure to 1-propanol would be primarily through the oral route via consumption of agricultural crops. The Environmental Health Criteria Monographs of the World Health Organization International Programme on Chemical Safety comment that exposure of the general population to 1-propanol is via drinking water, alcoholic and non-alcoholic beverages, and food stuffs [3].

Oral LD50 values for 1-propanol range between 1870 and $6800 \mathrm{mg} / \mathrm{kg}$ body weight in several animal species. The dermal LD50 is $4000-6700 \mathrm{mg} / \mathrm{kg}$ in rabbits. In a skin irritation study, mild effects (well-defined erythema and slight

Cheol-Hong Lim

limch@kosha.or.kr

1 Chemical Research Bureau, Occupational Safety and Health Research Institute, KOSHA, Daejeon 34122, Republic of Korea edema) were observed in rabbits [2]. In an acute inhalation study, rats were exposed to 1-propanol vapors for $4 \mathrm{~h}$ at a concentration of $9840 \mathrm{mg} / \mathrm{m}^{3}$ (3936 ppm), and two out of six rats died within 14 days [3]. In a limited oral repeated dose study in male rats (1-propanol in drinking water for 4 months), the no-observed-adverse-effect level (NOAEL) was $3000 \mathrm{mg} / \mathrm{kg}$ body weight [4]. 1-Propanol has not shown mutagenic potential [2].

Because of its relatively low toxicity, 1-propanol is not considered harmful by food or non-occupational exposures. However, from an occupational perspective, workers are potentially exposed by inhalation to 1-propanol during the production of the compound or its derivatives, or during its use in solvent-type applications. Thus, additional inhalation toxicity data are needed to assess the hazard of 1-propanol for workers safety. Therefore, we performed a 4-week repeated inhalation study in rats to obtain data on the toxic effects of 1-propanol by inhalation exposure.

\section{Materials and methods}

\section{Test material and animals}

1-Propanol (99.9\%) was purchased from Duksan Pure Chemicals (Ansan, Korea). Six-week-old specific-pathogen-free 
Fischer 344 (F344) rats were purchased from Japan SLC (Tokyo, Japan) and acclimatized for 7 days before initial exposure. Rats were housed in an animal room and inhalation chambers that were maintained at $22 \pm 3{ }^{\circ} \mathrm{C}$ and $50 \pm 20 \%$ relative humidity with a $12 \mathrm{~h}$ light/dark cycle. The chambers were vented 12 times per hour and oxygen concentrations were maintained at a minimum of $19 \%$ during the exposure period. Rats were fed $18 \%$ protein Rodent Diet 2918C (Envigo RMS, Indianapolis, IN, USA) and had access to water ad libitum. During the exposure period, rats were housed individually in a multi-compartment stainless steel wire mesh cage $(\mathrm{W} 240 \times \mathrm{L} 1200 \times \mathrm{H} 200 \mathrm{~mm})$ in the WITC-14 M whole-body inhalation chamber (HCT, Icheon, Korea). Groups of five rats of each sex were exposed to 0 , 100, 400, and 1600 ppm 1-propanol, 6 h/day, 5 days/week, for 4 weeks. The study was approved by the Institutional Animal Care and Use Committee at the Chemical Research Bureau, Occupational Safety and Health Research Institute prior to obtaining the rats. All experiments were conducted in accordance with established animal care protocols.

\section{Test material generation and analysis}

1-Propanol was converted to a vapor by a LVG-04-A liquid vapor generator (HCT). An IRGAS Fourier-transform infrared spectrometer (Lab Frontier, Anyang, Korea) was used to analyze the concentrations of 1-propanol in the inhalation chambers. Vapors were diluted by regulating clean air flow through the liquid reservoir container of 1-propanol in the generating system. Analytical chamber concentrations were measured at least three times each exposure day. During the exposure period, test material in the liquid reservoir was replaced weekly.

\section{Clinical observations and mean body weight}

All animals were observed daily during the study period for mortality and the development of clinical signs including respiratory, dermal, behavioral, nasal, and genitourinary changes. Body weight was measured using an electronic balance (Quintix 3102, Sartorius, Göttingen, Germany) twice a week for the study period.

\section{Hematology and blood biochemistry}

After the 4-week exposure, all rats were fasted overnight and blood samples were collected from the abdominal aorta after anesthesia with isoflurane (Il-sung Pharm, Seoul, Korea). Hematological parameters, including the total red blood cell count, hematocrit (HCT), hemoglobin concentration, mean corpuscular volume, mean corpuscular hemoglobin, mean corpuscular hemoglobin concentration (MCHC), platelet count, reticulocyte (RET) count, total white blood cell count, differential white blood cell count (neutrophils, lymphocytes, monocytes, eosinophils, and basophils), activated partial thromboplastin time (APTT), and prothrombin time were determined with an ADIVA 2120i blood cell analyzer (Siemens Diagnostics, Tarrytown, NY, USA) or an ACL ELITE Systems coagulation analyzer (Instrumentation Laboratory, Bedford, MA, USA). Blood biochemical parameters, such as alanine aminotransferase, aspartate aminotransferase, alkaline phosphatase (ALP), total protein, albumin, triglyceride, total cholesterol (TCHO), total bilirubin, blood urea nitrogen, creatinine, creatinine phosphokinase, sodium, and potassium, were measured with a TBA-120FR blood chemistry analyzer (Toshiba, Tokyo, Japan).

\section{Necropsy, organ weights, and histopathological examination}

All rats underwent a complete necropsy that involved examining the external body surfaces and all orifices, as well as the cranial, thoracic, and abdominal cavities and their contents. The following organs were trimmed and weighed: adrenal glands, brain, heart, kidneys, liver, lung, spleen, testes, thymus, epididymides, ovaries, and uterus. In addition, the bone marrow, esophagus, femur, stifle joint, larynx, lymph nodes (tracheobronchial and mesenteric), nasopharyngeal tissue, ovaries, seminal vesicles, spinal cord (cervical, lumbar, and thoracic), stomach, thyroid, trachea, and urinary bladder were collected and preserved in 10\% neutral buffered formalin. The eye/optic nerves and testes were collected and preserved in Davison's solution. All preserved tissues from male and female rats of the control $(0 \mathrm{ppm})$ and high dose (1600 ppm) groups were paraffinembedded, sectioned, stained with hematoxylin and eosin, and examined microscopically.

\section{Statistical analysis}

Data obtained during the study period are presented as mean \pm standard deviation. Data were assumed to be normally distributed and analyzed by a one-way analysis of variance. Dunnett's multiple comparison test was used as the post hoc test. SPSS v18 (IBM, Chicago, IL, USA) or SigmaPlot v12 (Systat Software, San Jose, CA, USA) were used for all statistical analyses. A $p$ value $<0.05$ was considered as significant.

\section{Results}

\section{Exposure and analysis of the test material}

Concentrations of 1-propanol in the exposure chamber during the study period for the low (100 ppm), 
middle (400 ppm), and high (1600 ppm) dose groups were $100.11 \pm 5.10 \mathrm{ppm}, 403.19 \pm 12.31 \mathrm{ppm}$, and $1598.08 \pm 139.58$ ppm, respectively (Fig. 1).

\section{Clinical signs and mean body weight changes}

No exposure-related clinical signs or changes in food consumption (data not shown), or significant differences of mean body weights (Fig. 2) were observed during the study period.

\section{Hematology and blood biochemistry}

No significant changes were noted in the hematology results between the control and different dose groups in female rats. However, in male rats, the MCHC was increased $(p<0.05)$ in the low (100 ppm) dose group, and APTT was increased $(p<0.05)$ in the middle $(400 \mathrm{ppm})$ and high $(1600 \mathrm{ppm})$ dose groups. On the other hand, RETs were decreased $(p<0.05)$ in the middle dose group (Table 1). The blood biochemistry results showed an increased TCHO $(p<0.05)$ in males in the low and middle dose groups, and increased sodium $(p<0.05)$ and chloride $(p<0.05)$ in the middle and

Fig. 1 Concentration of 1-Propanol exposed to $0 \mathrm{ppm}$, $100 \mathrm{ppm}, 400 \mathrm{ppm}$, and $1600 \mathrm{ppm}$ during the exposure period
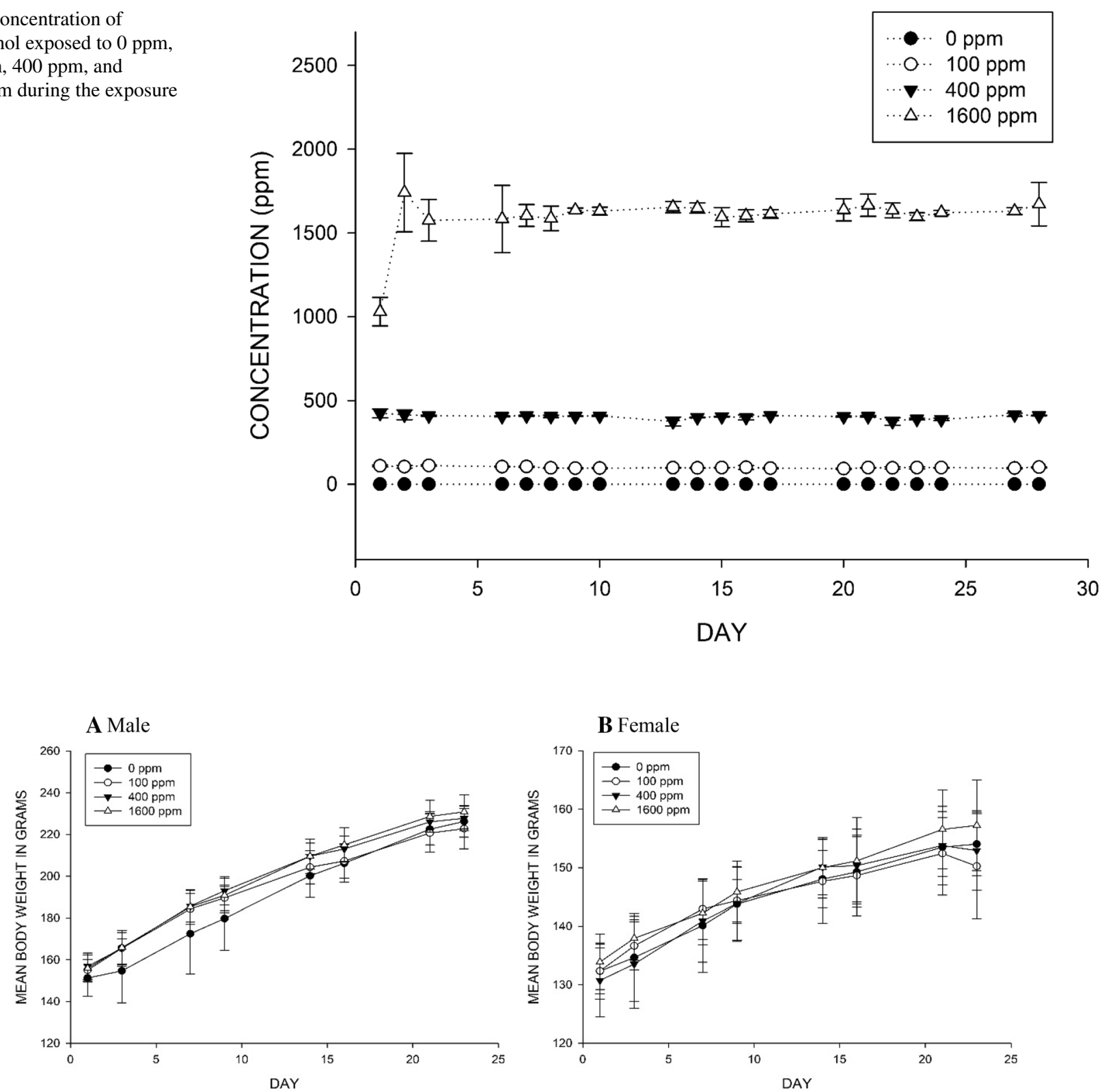

Fig. 2 Mean body weight of rats exposed to 0 ppm, 100 ppm, $400 \mathrm{ppm}$ and $1600 \mathrm{ppm}$ of 1-Propanol during the experimental period 
Table 1 Hematological values of male and female rats in the 4-week inhalation toxicity study of 1-Propanol

\begin{tabular}{|c|c|c|c|c|}
\hline Parameters & Control (0 ppm) & Low (100 ppm) & Middle (400 ppm) & High (1600 ppm) \\
\hline \multicolumn{5}{|l|}{ Male } \\
\hline $\mathrm{RBC}\left(\times 10^{6} / \mu \mathrm{L}\right)$ & $8.47 \pm 0.30$ & $8.64 \pm 0.19$ & $8.73 \pm 0.22$ & $8.71 \pm 0.22$ \\
\hline $\operatorname{HCT}(\%)$ & $43.5 \pm 1.1$ & $43.7 \pm 1.0$ & $44.4 \pm 1.0$ & $44.2 \pm 1.2$ \\
\hline HGB $(\mathrm{g} / \mathrm{dL})$ & $14.9 \pm 0.4$ & $15.3 \pm 0.3$ & $15.3 \pm 0.2$ & $15.4 \pm 0.4$ \\
\hline MCV (fL) & $51.3 \pm 1.0$ & $50.6 \pm 0.2$ & $50.8 \pm 0.5$ & $50.7 \pm 1.0$ \\
\hline $\mathrm{MCH}(\mathrm{pg})$ & $17.6 \pm 0.4$ & $17.7 \pm 0.2$ & $17.5 \pm 0.3$ & $17.7 \pm 0.4$ \\
\hline $\mathrm{MCHC}(\mathrm{g} / \mathrm{dL})$ & $34.2 \pm 0.3$ & $35.0 \pm 0.3^{*}$ & $34.4 \pm 0.5$ & $34.9 \pm 0.2$ \\
\hline $\operatorname{PLT}\left(\times 10^{3} / \mu \mathrm{L}\right)$ & $819 \pm 48$ & $776 \pm 26$ & $762 \pm 52$ & $803 \pm 63$ \\
\hline $\operatorname{RET}\left(\times 10^{3} / \mu \mathrm{L}\right)$ & $247.6 \pm 34.9$ & $192.2 \pm 19.0$ & $177.0 \pm 20.2^{*}$ & $204.6 \pm 25.8$ \\
\hline $\operatorname{WBC}\left(\times 10^{3} / \mu \mathrm{L}\right)$ & $4.13 \pm 0.59$ & $4.89 \pm 1.23$ & $4.21 \pm 1.12$ & $3.89 \pm 0.63$ \\
\hline $\mathrm{NEU}\left(\times 10^{3} / \mu \mathrm{L}\right)$ & $1.07 \pm 0.23$ & $1.06 \pm 0.23$ & $1.00 \pm 0.17$ & $1.04 \pm 0.16$ \\
\hline $\operatorname{LYM}\left(\times 10^{3} / \mu \mathrm{L}\right)$ & $2.91 \pm 0.60$ & $3.65 \pm 0.99$ & $3.04 \pm 0.97$ & $2.69 \pm 0.57$ \\
\hline $\operatorname{MON}\left(\times 10^{3} / \mu \mathrm{L}\right)$ & $0.08 \pm 0.01$ & $0.08 \pm 0.02$ & $0.08 \pm 0.02$ & $0.07 \pm 0.01$ \\
\hline $\operatorname{EOS}\left(\times 10^{3} / \mu \mathrm{L}\right)$ & $0.04 \pm 0.01$ & $0.06 \pm 0.01$ & $0.05 \pm 0.01$ & $0.05 \pm 0.01$ \\
\hline BAS $\left(\times 10^{3} / \mu \mathrm{L}\right)$ & $0.01 \pm 0.01$ & $0.01 \pm 0.00$ & $0.00 \pm 0.01$ & $0.00 \pm 0.01$ \\
\hline APTT (sec) & $16.8 \pm 1.0$ & $18.2 \pm 0.8$ & $18.5 \pm 0.5^{*}$ & $18.4 \pm 0.5^{*}$ \\
\hline PT (sec) & $10.4 \pm 0.2$ & $10.4 \pm 0.2$ & $11.0 \pm 0.4$ & $10.3 \pm 0.3$ \\
\hline \multicolumn{5}{|l|}{ Female } \\
\hline $\mathrm{RBC}\left(\times 10^{6} / \mu \mathrm{L}\right)$ & $8.59 \pm 0.22$ & $8.89 \pm 0.16$ & $8.49 \pm 0.31$ & $8.56 \pm 0.20$ \\
\hline $\operatorname{HCT}(\%)$ & $44.1 \pm 1.2$ & $45.4 \pm 0.8$ & $43.8 \pm 1.8$ & $44.3 \pm 0.5$ \\
\hline HGB (g/dL) & $15.5 \pm 0.4$ & $16.1 \pm 0.2$ & $15.3 \pm 0.8$ & $15.6 \pm 0.4$ \\
\hline MCV (fL) & $51.4 \pm 0.3$ & $51.0 \pm 0.4$ & $51.6 \pm 0.8$ & $51.7 \pm 1.0$ \\
\hline $\mathrm{MCH}(\mathrm{pg})$ & $18.1 \pm 0.1$ & $18.1 \pm 0.1$ & $18.1 \pm 0.3$ & $18.2 \pm 0.1$ \\
\hline $\mathrm{MCHC}(\mathrm{g} / \mathrm{dL})$ & $35.2 \pm 0.2$ & $35.4 \pm 0.3$ & $35.1 \pm 0.9$ & $35.3 \pm 0.6$ \\
\hline $\operatorname{PLT}\left(\times 10^{3} / \mu \mathrm{L}\right)$ & $813 \pm 36$ & $794 \pm 96$ & $773 \pm 92$ & $809 \pm 110$ \\
\hline $\operatorname{RET}\left(\times 10^{3} / \mu \mathrm{L}\right)$ & $187.8 \pm 12.6$ & $199.9 \pm 14.0$ & $188.5 \pm 52.6$ & $198.4 \pm 20.5$ \\
\hline $\mathrm{WBC}\left(\times 10^{3} / \mu \mathrm{L}\right)$ & $3.94 \pm 0.67$ & $4.03 \pm 1.53$ & $4.27 \pm 1.40$ & $3.99 \pm 0.77$ \\
\hline $\mathrm{NEU}\left(\times 10^{3} / \mu \mathrm{L}\right)$ & $0.97 \pm 0.28$ & $1.00 \pm 0.64$ & $0.99 \pm 0.26$ & $0.93 \pm 0.30$ \\
\hline $\mathrm{LYM}\left(\times 10^{3} / \mu \mathrm{L}\right)$ & $2.77 \pm 0.53$ & $2.86 \pm 0.81$ & $3.08 \pm 1.05$ & $2.86 \pm 0.49$ \\
\hline $\operatorname{MON}\left(\times 10^{3} / \mu \mathrm{L}\right)$ & $0.09 \pm 0.03$ & $0.07 \pm 0.04$ & $0.10 \pm 0.04$ & $0.10 \pm 0.04$ \\
\hline $\operatorname{EOS}\left(\times 10^{3} / \mu \mathrm{L}\right)$ & $0.06 \pm 0.00$ & $0.06 \pm 0.03$ & $0.06 \pm 0.03$ & $0.06 \pm 0.02$ \\
\hline BAS $\left(\times 10^{3} / \mu \mathrm{L}\right)$ & $0.01 \pm 0.01$ & $0.01 \pm 0.01$ & $0.01 \pm 0.00$ & $0.01 \pm 0.01$ \\
\hline APTT (sec) & $18.2 \pm 1.1$ & $18.4 \pm 2.1$ & $16.2 \pm 1.5$ & $19.4 \pm 0.7$ \\
\hline PT (sec) & $10.8 \pm 0.5$ & $10.6 \pm 0.9$ & $11.1 \pm 0.2$ & $11.4 \pm 0.4$ \\
\hline
\end{tabular}

All values are expressed as mean \pm standard deviation. Significantly different from control $(* p<0.05)$ APTT activated partial thromboplastin time, BAS basophil, EOS eosinophil, $H C T$ hematocrit, $H G B$ hemoglobin, $L Y M$ lymphocyte, $M C H$ mean corpuscular hemoglobin, $M C H C$ mean corpuscular hemoglobin concentration, $M C V$ mean corpuscular volume, $M O N$ monocyte, $N E U$ neutrophil, PLT platelet, PT prothrombin time, $R B C$ red blood cell count, $R E T$ reticulocyte, $W B C$ white blood cell count high dose groups. In addition, decreased ALP $(p<0.05)$ was observed in males in the middle dose group. In females, decreased potassium $(p<0.05)$ in the middle dose group was the only change observed (Table 2 ).

\section{Organ weights, and histopathological examination}

There was a significant decrease in the absolute testes weight $(p<0.05)$ in males at the low $(100 \mathrm{ppm})$ dose compared to the male control group. The relative liver weight was significantly decreased $(p<0.05)$ in males at the low dose compared to male controls. Other organ weights did not show significant changes in either sex (Tables 3, 4). Mononuclear cell infiltration in heart, liver, and lung, metaplasia, macrophage aggregates in lung bronchi, and intracytoplasmic hyaline inclusions in the trachea were observed in male and female rats. In addition, tubule basophilia in the kidney and submucosal gland dilation in the trachea were observed in male rats, and focal nephropathy in the kidney was observed in female rats (Table 5). 
Table 2 Blood biochemical values of male and female rats in the 4-week inhalation toxicity study of 1-Propanol

\begin{tabular}{|c|c|c|c|c|}
\hline Parameters & Control (0 ppm) & Low (100 ppm) & Middle (400 ppm) & High (1600 ppm) \\
\hline \multicolumn{5}{|l|}{ Male } \\
\hline ALT (IU/L) & $35.2 \pm 2.2$ & $37.3 \pm 2.2$ & $36.6 \pm 1.5$ & $36.5 \pm 1.6$ \\
\hline AST (IU/L) & $77.6 \pm 7.6$ & $77.2 \pm 7.6$ & $79.7 \pm 4.1$ & $81.4 \pm 9.1$ \\
\hline ALP (IU/L) & $736.7 \pm 34.6$ & $679.9 \pm 34.6$ & $620.0 \pm 38.9^{*}$ & $726.9 \pm 83.8$ \\
\hline $\mathrm{TP}(\mathrm{g} / \mathrm{dL})$ & $5.7 \pm 0.0$ & $5.6 \pm 0.1$ & $5.7 \pm 0.3$ & $5.8 \pm 0.1$ \\
\hline ALB $(g / d L)$ & $4.1 \pm 0.0$ & $4.0 \pm 0.1$ & $4.1 \pm 0.1$ & $4.1 \pm 0.1$ \\
\hline $\mathrm{TG}(\mathrm{mg} / \mathrm{dL})$ & $56.4 \pm 14.4$ & $71.0 \pm 21.7$ & $55.3 \pm 10.3$ & $66.5 \pm 26.2$ \\
\hline TCHO (mg/dL) & $59.8 \pm 1.6$ & $78.6 \pm 11.8^{*}$ & $75.3 \pm 10.1^{*}$ & $69.6 \pm 11.2$ \\
\hline TBIL (mg/dL) & $0.16 \pm 0.02$ & $0.16 \pm 0.02$ & $0.15 \pm 0.01$ & $0.15 \pm 0.01$ \\
\hline BUN (mg/dL) & $20.3 \pm 2.6$ & $20.3 \pm 1.5$ & $20.1 \pm 1.6$ & $21.0 \pm 2.6$ \\
\hline CREA (mg/dL) & $0.38 \pm 0.03$ & $0.38 \pm 0.02$ & $0.40 \pm 0.03$ & $0.39 \pm 0.01$ \\
\hline CPK (U/L) & $325.8 \pm 112.6$ & $299.2 \pm 151.0$ & $337.6 \pm 104.1$ & $366.9 \pm 175.2$ \\
\hline $\mathrm{Na}(\mathrm{mmol} / \mathrm{L})$ & $140.5 \pm 4.0$ & $146.2 \pm 1.2$ & $147.0 \pm 1.0^{*}$ & $146.7 \pm 0.4^{*}$ \\
\hline $\mathrm{Cl}(\mathrm{mmol} / \mathrm{L})$ & $97.9 \pm 0.6$ & $100.0 \pm 1.1$ & $100.6 \pm 1.4^{*}$ & $101.2 \pm 0.8^{*}$ \\
\hline $\mathrm{K}(\mathrm{mmol} / \mathrm{L})$ & $4.16 \pm 0.69$ & $4.64 \pm 0.25$ & $4.61 \pm 0.25$ & $4.74 \pm 0.18$ \\
\hline \multicolumn{5}{|l|}{ Female } \\
\hline ALT (IU/L) & $38.2 \pm 4.1$ & $40.1 \pm 6.2$ & $33.7 \pm 2.5$ & $40.9 \pm 5.0$ \\
\hline AST (IU/L) & $96.0 \pm 19.2$ & $90.9 \pm 10.2$ & $85.9 \pm 7.6$ & $103.8 \pm 36.0$ \\
\hline ALP (IU/L) & $543.1 \pm 12.2$ & $530.2 \pm 20.4$ & $519.8 \pm 31.2$ & $506.6 \pm 38.7$ \\
\hline $\mathrm{TP}(\mathrm{g} / \mathrm{dL})$ & $5.5 \pm 0.2$ & $5.5 \pm 0.2$ & $5.7 \pm 0.1$ & $6.8 \pm 1.9$ \\
\hline $\operatorname{ALB}(g / d L)$ & $4.0 \pm 0.1$ & $4.0 \pm 0.1$ & $4.1 \pm 0.1$ & $4.2 \pm 0.1$ \\
\hline $\mathrm{TG}(\mathrm{mg} / \mathrm{dL})$ & $14.6 \pm 4.0$ & $18.2 \pm 2.8$ & $11.1 \pm 0.5$ & $13.3 \pm 6.2$ \\
\hline TCHO (mg/dL) & $70.6 \pm 3.6$ & $70.1 \pm 4.0$ & $64.1 \pm 11.9$ & $68.7 \pm 6.1$ \\
\hline TBIL (mg/dL) & $0.15 \pm 0.01$ & $0.15 \pm 0.01$ & $0.16 \pm 0.02$ & $0.12 \pm 0.07$ \\
\hline BUN (mg/dL) & $20.4 \pm 3.1$ & $18.8 \pm 1.4$ & $20.0 \pm 3.7$ & $22.1 \pm 1.7$ \\
\hline CREA (mg/dL) & $0.37 \pm 0.02$ & $0.37 \pm 0.02$ & $0.39 \pm 0.02$ & $0.37 \pm 0.04$ \\
\hline CPK (U/L) & $377.3 \pm 161.3$ & $281.8 \pm 129.3$ & $286.3 \pm 109.5$ & $463.2 \pm 353.2$ \\
\hline $\mathrm{Na}(\mathrm{mmol} / \mathrm{L})$ & $146.0 \pm 1.6$ & $147.3 \pm 0.9$ & $147.4 \pm 0.6$ & $146.7 \pm 1.2$ \\
\hline $\mathrm{Cl}(\mathrm{mmol} / \mathrm{L})$ & $102.0 \pm 0.7$ & $102.7 \pm 0.7$ & $102.6 \pm 0.5$ & $103.0 \pm 0.9$ \\
\hline $\mathrm{K}(\mathrm{mmol} / \mathrm{L})$ & $4.50 \pm 0.11$ & $4.45 \pm 0.13$ & $3.96 \pm 0.30^{*}$ & $4.58 \pm 1.13$ \\
\hline
\end{tabular}

All values are expressed as mean \pm standard deviation. Significantly different from control $\left({ }^{*} p<0.05\right)$

$A L B$ albumin, $A L P$ alkaline phophatase, $A L T$ alanine aminotransferase, $A S T$ aspartate aminotransferase, $B U N$ blood urea nitrogen, $C P K$ creatine phosphokinase, $C R E A$ creatinine, $K$ potassium, $N a$ sodium, $T B I L$ total bilirubin, $T C H O$ total cholesterol, $T G$ triglyceride, $T P$ total protein

\section{Discussion}

1-Propanol is used by consumers (lubricants, coating products, anti-freeze products, sealants, polishes, cosmetics, and others), by professional workers (metal working fluids, scientific research, health services, electrical, optical equipment, and others), in formulation or re-packing, at industrial sites, and in manufacturing. 1-Propanol is manufactured and imported into the European Economic Area at 10,000 - 100,000 tons per year [5]. It is generally considered to be a relatively safe substance because its exposure is mainly oral and it exhibits low toxicity. However, studies and assessments of inhalation exposures, the main route of workers, are inadequate. For this reason, we performed a 4-week repeated inhalation toxicity study according to the Organisation for Economic Co-operation and
Development Guidelines for the Testing of Chemicals [6]. Concentrations of 0, 100, 400, and $1600 \mathrm{ppm}$ were selected for the control, low, middle, and high dose groups, based on a previous report that two out of six animals died within 14 days after a 1-propanol exposure at $3936 \mathrm{ppm}$ for $4 \mathrm{~h}$ [3].

In the current study, F344 rats were exposed to 1-propanol vapor $6 \mathrm{~h}$ a day, 5 days per week, for 4 weeks. No mortality or clinical signs related to this exposure were observed. Furthermore, there were no significant changes in body weight or food consumption during the 4-week exposure period. However, a relatively high standard deviation and unstable mean body weight were observed during the study period. This was considered to be caused by the stress of housing in stainless steel wire mesh cages during the exposure period, resulting in individual differences between animals. 
Table 3 Absolute organ weight of male and female rats in the 4-week inhalation toxicity study of 1-Propanol (Unit: g)
Table 4 Relative organ weight of male and female rats in the 4-week inhalation toxicity study of 1-Propanol (Unit: g)

\begin{tabular}{lllll}
\hline Organs & Control $(0 \mathrm{ppm})$ & Low $(100 \mathrm{ppm})$ & Middle $(400 \mathrm{ppm})$ & High $(1600 \mathrm{ppm})$ \\
\hline Male & & & & \\
Adrenal glands & $0.043 \pm 0.004$ & $0.043 \pm 0.001$ & $0.043 \pm 0.003$ & $0.043 \pm 0.002$ \\
Brain & $1.781 \pm 0.030$ & $1.754 \pm 0.052$ & $1.817 \pm 0.017$ & $1.779 \pm 0.046$ \\
Epididymides & $0.627 \pm 0.053$ & $0.542 \pm 0.087$ & $0.598 \pm 0.030$ & $0.620 \pm 0.055$ \\
Heart & $0.647 \pm 0.042$ & $0.637 \pm 0.015$ & $0.641 \pm 0.023$ & $0.659 \pm 0.027$ \\
Kidneys & $1.473 \pm 0.064$ & $1.379 \pm 0.042$ & $1.445 \pm 0.079$ & $1.490 \pm 0.064$ \\
Liver & $6.754 \pm 0.443$ & $6.108 \pm 0.477$ & $6.248 \pm 0.258$ & $6.544 \pm 0.175$ \\
Lung & $0.905 \pm 0.014$ & $0.887 \pm 0.025$ & $0.878 \pm 0.027$ & $0.908 \pm 0.027$ \\
Spleen & $0.535 \pm 0.036$ & $0.474 \pm 0.033$ & $0.479 \pm 0.032$ & $0.498 \pm 0.040$ \\
Testes & $2.625 \pm 0.055$ & $2.390 \pm 0.211^{*}$ & $2.549 \pm 0.104$ & $2.596 \pm 0.058$ \\
Thymus & $0.266 \pm 0.021$ & $0.301 \pm 0.017$ & $0.286 \pm 0.018$ & $0.279 \pm 0.030$ \\
Female & & & & \\
Adrenal glands & $0.050 \pm 0.006$ & $0.050 \pm 0.004$ & $0.049 \pm 0.006$ & $0.051 \pm 0.006$ \\
Brain & $1.674 \pm 0.033$ & $1.654 \pm 0.048$ & $1.666 \pm 0.050$ & $1.680 \pm 0.029$ \\
Heart & $0.499 \pm 0.029$ & $0.491 \pm 0.022$ & $0.508 \pm 0.031$ & $0.503 \pm 0.024$ \\
Kidneys & $1.051 \pm 0.070$ & $1.046 \pm 0.047$ & $1.056 \pm 0.056$ & $1.062 \pm 0.043$ \\
Liver & $3.867 \pm 0.139$ & $3.804 \pm 0.157$ & $3.876 \pm 0.280$ & $3.986 \pm 0.234$ \\
Lung & $0.736 \pm 0.043$ & $0.729 \pm 0.044$ & $0.712 \pm 0.025$ & $0.760 \pm 0.026$ \\
Ovaries & $0.060 \pm 0.016$ & $0.065 \pm 0.012$ & $0.062 \pm 0.010$ & $0.066 \pm 0.008$ \\
Spleen & $0.365 \pm 0.014$ & $0.359 \pm 0.033$ & $0.357 \pm 0.018$ & $0.376 \pm 0.019$ \\
Thymus & $0.247 \pm 0.010$ & $0.248 \pm 0.026$ & $0.234 \pm 0.019$ & $0.250 \pm 0.020$ \\
Uterus & $0.431 \pm 0.241$ & $0.251 \pm 0.027$ & $0.334 \pm 0.052$ & $0.303 \pm 0.031$ \\
\hline All valyes & & & \\
\hline
\end{tabular}

All values are expressed as mean \pm standard deviation

Significantly different from control $(* p<0.05)$

\begin{tabular}{lllll}
\hline Organs & Control $(0 \mathrm{ppm})$ & Low $(100 \mathrm{ppm})$ & Middle $(400 \mathrm{ppm})$ & High $(1600 \mathrm{ppm})$ \\
\hline Male & & & \\
Adrenal glands & $0.020 \pm 0.002$ & $0.020 \pm 0.001$ & $0.020 \pm 0.002$ & $0.020 \pm 0.001$ \\
Brain & $0.817 \pm 0.033$ & $0.825 \pm 0.045$ & $0.848 \pm 0.035$ & $0.811 \pm 0.020$ \\
Epididymides & $0.287 \pm 0.024$ & $0.254 \pm 0.034$ & $0.279 \pm 0.011$ & $0.283 \pm 0.022$ \\
Heart & $0.297 \pm 0.018$ & $0.299 \pm 0.006$ & $0.299 \pm 0.013$ & $0.300 \pm 0.011$ \\
Kidneys & $0.675 \pm 0.012$ & $0.656 \pm 0.008$ & $0.674 \pm 0.022$ & $0.679 \pm 0.034$ \\
Liver & $3.093 \pm 0.125$ & $2.864 \pm 0.129^{*}$ & $2.913 \pm 0.034$ & $2.987 \pm 0.057$ \\
Lung & $0.415 \pm 0.014$ & $0.416 \pm 0.009$ & $0.410 \pm 0.016$ & $0.414 \pm 0.014$ \\
Spleen & $0.245 \pm 0.016$ & $0.222 \pm 0.009$ & $0.223 \pm 0.013$ & $0.227 \pm 0.013$ \\
Testes & $1.204 \pm 0.048$ & $1.121 \pm 0.078$ & $1.189 \pm 0.036$ & $1.184 \pm 0.037$ \\
Thymus & $0.122 \pm 0.010$ & $0.142 \pm 0.012$ & $0.133 \pm 0.010$ & $0.127 \pm 0.010$ \\
Female & & & & \\
Adrenal glands & $0.035 \pm 0.003$ & $0.036 \pm 0.003$ & $0.035 \pm 0.003$ & $0.035 \pm 0.004$ \\
Brain & $1.186 \pm 0.050$ & $1.180 \pm 0.030$ & $1.180 \pm 0.080$ & $1.160 \pm 0.080$ \\
Heart & $0.354 \pm 0.030$ & $0.350 \pm 0.010$ & $0.360 \pm 0.020$ & $0.350 \pm 0.010$ \\
Kidneys & $0.744 \pm 0.040$ & $0.075 \pm 0.030$ & $0.740 \pm 0.040$ & $0.730 \pm 0.020$ \\
Liver & $2.739 \pm 0.110$ & $2.710 \pm 0.060$ & $2.730 \pm 0.210$ & $2.750 \pm 0.100$ \\
Lung & $0.521 \pm 0.026$ & $0.521 \pm 0.024$ & $0.502 \pm 0.015$ & $0.526 \pm 0.038$ \\
Ovaries & $0.042 \pm 0.011$ & $0.047 \pm 0.009$ & $0.044 \pm 0.007$ & $0.046 \pm 0.006$ \\
Spleen & $0.258 \pm 0.007$ & $0.256 \pm 0.017$ & $0.252 \pm 0.010$ & $0.260 \pm 0.011$ \\
Thymus & $0.175 \pm 0.006$ & $0.177 \pm 0.020$ & $0.164 \pm 0.011$ & $0.172 \pm 0.007$ \\
Uterus & $0.301 \pm 0.158$ & $0.179 \pm 0.022$ & $0.235 \pm 0.028$ & $0.209 \pm 0.012$ \\
\hline Allvaly & & & \\
\hline
\end{tabular}

All values are expressed as mean \pm standard deviation

Significantly different from control $\left({ }^{*} p<0.05\right)$ 
Table 5 Histopathological findings of male and female rats in the 4-week inhalation toxicity study of 1-Propanol

\begin{tabular}{|c|c|c|}
\hline Histopathological findings & $0 \mathrm{ppm}$ & $1600 \mathrm{ppm}$ \\
\hline \multicolumn{3}{|l|}{ Male } \\
\hline \multicolumn{3}{|l|}{ Heart } \\
\hline Infiltration, mononuclear cell & $1 / 5$ & $2 / 5$ \\
\hline \multicolumn{3}{|l|}{ Kidney } \\
\hline Basophilia, tubules & $1 / 5$ & $0 / 5$ \\
\hline \multicolumn{3}{|l|}{ Liver } \\
\hline Infiltration, mononuclear cell & $1 / 5$ & $0 / 5$ \\
\hline \multicolumn{3}{|l|}{ Lung with bronchi } \\
\hline Metaplasia, osseous & $0 / 5$ & $1 / 5$ \\
\hline Infiltration, mononuclear cell, subpleural & $2 / 5$ & $4 / 5$ \\
\hline Aggregate, macrophage, alveolar & $3 / 5$ & $2 / 5$ \\
\hline \multicolumn{3}{|l|}{ Trachea } \\
\hline Dilation, submucosal gland & $1 / 5$ & $0 / 5$ \\
\hline Intracytoplasmic hyaline inclusion & $3 / 5$ & $5 / 5$ \\
\hline \multicolumn{3}{|l|}{ Female } \\
\hline \multicolumn{3}{|l|}{ Heart } \\
\hline Infiltration, mononuclear cell & $0 / 5$ & $1 / 5$ \\
\hline \multicolumn{3}{|l|}{ Kidney } \\
\hline Focal nephropathy, bilateral & $0 / 5$ & $1 / 5$ \\
\hline Calculi & $1 / 5$ & $2 / 5$ \\
\hline \multicolumn{3}{|l|}{ Liver } \\
\hline Infiltration, mononuclear cell & $2 / 5$ & $2 / 5$ \\
\hline \multicolumn{3}{|l|}{ Lung with bronchi } \\
\hline Metaplasia, osseous & $0 / 5$ & $1 / 5$ \\
\hline Infiltration, mononuclear cell, subpleural & $2 / 5$ & $1 / 5$ \\
\hline Aggregate, macrophage, alveolar & $0 / 5$ & $1 / 5$ \\
\hline \multicolumn{3}{|l|}{ Trachea } \\
\hline Intracytoplasmic hyaline inclusion & $3 / 5$ & $1 / 5$ \\
\hline
\end{tabular}

Data was presented as incidence with examined animals

The hematology results revealed increased MCHC, APTT, and decreased RETs. MCHC is used to define the concentration of hemoglobin mathematically and to suggest the restoration of oxygen carrying capacity of the blood, while reduced RETs is indicative of bone marrow suppression [7]. However, there was no dose-response relationship, and changes in other related hematological markers were not observed. Generally, prolonged APTT and a normal prothrombin time could be interpreted as an artifact of sample collection or an indication of liver failure. However, there were no other changes in markers related to liver failure.

The blood biochemistry results showed increased TCHO, sodium, and chloride, and decreased ALP and potassium. TCHO typically reflects general metabolic events, and an increased or decreased TCHO may be related to changes in food consumption, body weight, liver function, and hormone balance. A lower serum ALP may be related to fasting or decreased food consumption. Increased sodium and chloride may reflect dehydration, and decreased potassium may be associated with prolonged decreased intake or gastrointestinal loss [8]. However, there was again no dose-response to these changes that remained within the normal range [9, 10]. In addition, we observed no changes of other related markers in this study.

The changes of absolute testes weight and relative liver weight did not occur in a dose-response manner, and there were no histopathological findings related to the test substance. Some microscopic findings observed in the control and high dose groups of male and female rats were considered to be incidental or spontaneous because they are observed commonly in similar aged rats as background lesions, and the frequency was similar to the results in control animals [11-13].

Based on these results, the NOAEL of 1-propanol is over $1600 \mathrm{ppm}$. This is quite different from what we expected based on existing literature [3] and the Material Safety Data Sheet (MSDS) provided by manufacturers of hazardous chemicals to inform users of any intrinsic toxic properties [14]. It is very important to provide correct information because the MSDS is widely used to protect workers' health in the work place. According to the latest European Chemicals Agency data, the LC50 of 1-propanol is 13,548 ppm [5]. The reason why the LC50 value provided by the International Programme on Chemical Safety and MSDS differs from that of the European Chemicals Agency is that the latter are from very old data and technical problems at that time may have affected the results. Thus, re-evaluating existing toxicity data and promptly applying the results of the re-evaluation are very important as science, technology, and new toxicity data develop. Determining the precise toxicity of 1-propanol via the inhalation route will require further studies. In conclusion, this study showed that there were no adverse effects in F344 rats following repeated inhalation exposure of 1-propanol during a 4-week period. Our findings suggest that the NOAEL of 1-propanol is over $1600 \mathrm{ppm}$.

\section{Compliance with ethical standards}

Conflict of interest The authors have no conflict of interest to disclose.

\section{References}

1. Vujasinović M, Koćar M, Kramer K, Bunc M, Brvar M (2007) Poisoning with 1-propanol and 2-propanol. Hum Exp Toxicol 26:975-978

2. United States Environmental Protection Agency (2005) Inert reassessment-n-propanol; CAS\# 71-23-8. Office of Prevention, Pesticides, and Toxic Substances. https://www.epa.gov/ingredient 
s-used-pesticide-products/inert-reassessment-document-n-propa nol-cas-no-71-23-8. Accessed 21 Jan 2019

3. International programme on chemical safety (IPCS) (1990) INCHEM, environmental health criteria 102 (EHC 102), 1-propanol. http://www.inchem.org/documents/ehc/ehc/ehc102.htm. Accessed 29 Jan 2019

4. European Chemicals Bureau (2008) European Union risk assessment report, propan-1-ol, vol 82. https://doi.org/10.2788/95030

5. European chemical agency, substance information, Propanol1-ol. https://ehca.europa.eu/substance-information/-/substancei nfo/100.000.679. Accessed 21 Jan 2019

6. Organisation for Economic Co-operation and Development (2009) Guideline for the testing of chemicals, section 4 health effects test No. 412 Subacute Inhalation Toxicity: 28-day study

7. Arika WM, Nyamai DW, Musila MN, Ngugi MP, Njagi ENM (2016) Hematological markers of in vivo toxicity. J Hematol Thrombo Dis 4:2

8. Wanda MH, Colin GR, Matthew AW (2013) Haschek and rousseaux's handbook of toxicologic pathology, 3rd edn. Acad Press, USA, pp 853-869

9. Matsuzawa T, Nomura M, Unno T (1993) Clinical pathology reference ranges of laboratory animals. J Vet Med Sci 55:351-362
10. Song CW, Kim HC, Kang BH, Han SS (1991) Studies on the basic data of Ktc: fisher (F344) rats with age-(1)-body weight, organ weight, hematology, serum biochemistry and urine analysis. Korean J Lab Ani Sci 7:22-33

11. Berridge BR, Mowat V, Nagai H, Nyska A, Okazaki Y, Clements PJ, Rinke M, Snyder PW, Boyle MC, Wells MY (2016) Nonproliferative and proliferative lesions of the cardiovascular system of the rat and mouse. J Toxicol Pathol 29:1S-47S. https://doi. org/10.1293/tox.29.3s-1

12. Frazier KS, Seely JC, Hard GC, Betton G, Burnett R, Nakatsuji S, Nishikawa A, Durchfeld-Meyer B, Bube A (2012) Proliferative and nonproliferative lesions of the rat and mouse urinary system. Toxicol Pathol 40:14S-86S. https://doi.org/10.1177/0192623312 438736

13. McInnes Elizabeth F, Mann Peter (2012) Background lesions in laboratory animals: a color Atlas. Saunders/Elsevier, Edinburgh, pp 20-22

14. Torres JA, Bobst S (2015) Toxicological risk assessment for beginners. Springer, Switzerland, pp 1160-1166 